\title{
Implicit versus explicit memory function in children with Down and Williams syndrome
}

\author{
Stefano Vicari, MD \\ IRCCS, Ospedale Pediatrico Bambino Gesù, Santa Marinella, Roma, Italy
}

\begin{abstract}
The present study was aimed at evaluating implicit memory processes in participants with Williams syndrome and comparing them to children with Down syndrome and to mental-age matched typically developing children. For this purpose, tests of verbal and visuo-perceptual explicit memory, verbal and visual repetition priming as well as procedural learning tasks were administered to 12 participants with Williams syndrome, 14 with Down syndrome and 32 typically developing children. Participants with Williams syndrome showed a level of repetition priming similar to that of mental-age typically developing controls. In contrast, children with Williams syndrome showed a reduced learning rate in the two procedural tasks. As regards children with Down syndrome, we document comparable implicit memory abilities. In contrast, regarding explicit memory, typically developing children performed better than individuals with Down syndrome. This finding is relevant for our knowledge about the qualitative aspects of the anomalous cognitive development in individuals with intellectual disabilities and the neurobiological substrate underlying this development.
\end{abstract}

Keywords - Implicit memory, learning, Williams syndrome, Down syndrome

\section{Introduction}

Neuropsychological research has allowed the defining of different cognitive profiles among participants with intellectual disabilities of different aetiology. For example, numerous authors have stressed that the typical language profile for persons with Down syndrome consists of poor production with greater compromising of morphosyntax than of lexical abilities, but relatively preserved comprehension (Miller, 1988; Miller, 1992; Vicari, Caselli \& Tonucci, 2000).

Williams syndrome is another genetic condition, less common but equally characterised by intellectual disabilities and typified by a number of severe medical anomalies, such as facial dysmorphology and abnormalities of the cardiovascular system (Ewart, Morris \& Atkinson, 1993; Frangiskakis, Ewart and Morris, 1996; Tassabehji, Karmiloff-Smith \& Grant, 1998; Tassabehji, Metcalfe and Fergusson, 1996; Botta, et al., 1999).

Children with Williams syndrome often show marked impairment in certain visual-spatial abilities (especially praxic-constructive) and relative preservation of both productive and receptive language, at least concerning the phonological elements (Volterra, Capirci, Pezzini, Sabbadini \& Vicari, 1996; Pezzini, Vicari, Volterra, Milani \& Ossella, 1999). Also different cognitive profiles were described in participants with comparable intellectual deficits or even with the same aetiology (Vicari, Albertini \& Caltagirone, 1992; Pezzini et al., 1999). All these observations seem to support a theoretical approach that considers intellectual disabilities not as a mere slowing of normal cognitive development, but as distinct, individual profiles, that can be qualitatively specified. In line with this theoretical point of view (which also suggests the need for strongly individualised teaching protocols), many recent studies emphasised the need to better define not only the impaired cognitive abilities in each individual, but just as importantly, the respective strengths, or relatively preserved abilities in children with intellectual disabilities. The importance of this approach was evidenced in several recent studies of memory, especially implicit memory in individuals with intellectual disabilities.

Neuropsychological studies reported in the literature suggest insufficient development of the memory function in intellectual disabilities. Though with some exceptions (for example, children with Williams syndrome, Vicari, Carlesimo, Brizzolara \& Pezzini, 1996) multiple deficits have been identified in short term memory functioning. The peripheral systems of articulatory rehearsal as well as the central systems that direct information processing seem to be deficient in these individuals (Hulme and Mackenzie, 1992).

Long-term memory has been also extensively investigated in persons with intellectual disabilities both in the explicit 
and in the implicit component. Explicit memory concerns intentional recalling or recognition of experiences or information. Implicit memory is manifested as a facilitation (that is an improvement in performance) in perceptual, cognitive and motor tasks, without any conscious reference to previous experiences. Explicit memory deficits in persons with intellectual disabilities have also been extensively documented. According to recent studies, due to this diffuse impairment of memory abilities, persons with intellectual disabilities should show a relative preservation of implicit memory.

In a recent study (Carlesimo, Marotta \& Vicari, 1997), we described long term memory abilities in persons with Down syndrome and in others with intellectual disabilities of unknown aetiology, comparing them with typically developing participants of similar mental age. The performance of the typically developing participants in explicit memory tests was significantly better than the children with intellectual disabilities of unknown aetiology, and the latter were better than those with Down syndrome. On the other hand, the performances of the three groups did not differ in an implicit memory test (repetition priming); both intellectual disabilities groups performed as well as mental age matched controls. These results seem to confirm a dissociation between explicit and implicit memory in individuals with intellectual disabilities. However, there are many limitations in the studies reported thus far on this issue: results are often contradictory and methodological limits include the use of populations with intellectual disabilities of often-undefined aetiology. Furthermore, the selection criteria used for the control group (chronological age, mental age), and the limited number of tests used for evaluating implicit memory (almost always visual priming tests) especially limit the conclusions which can be drawn. This last point is particularly interesting and has important implications for both theoretical and applied issues. Specifically, if the presumed facility demonstrated by persons with intellectual disabilities in repetition priming tests were confirmed, for example, in procedural learning tests, this would suggest substantial preservation of implicit memory functions, and thus would support the theoretical distinction between implicit and explicit memory. From a more applied prospective, these findings would suggest the possibility of using techniques based on automatic learning in the rehabilitation of these individuals.

In the present paper the results of two recent studies of our group carried out in our clinic in Santa Marinella are presented. They are concerned with different aspects of implicit and explicit memory in two groups of persons with intellectual disabilities (Down syndrome and Williams syndrome) compared with typically developing individuals matched for mental age, that is, with a comparable global cognitive level of functioning.
Our aims were, firstly, to verify the hypothesis that persons with intellectual disabilities have impaired explicit memory abilities compared with controls, but that the groups do not differ significantly in implicit memory abilities, and secondly, to find out if this profile is characteristic of all people with intellectual disabilities or, alternatively, whether distinct profiles may be described in different aetiological groups of intellectual disabilities.

\section{Methods and Materials}

\section{Participants}

The performance of three groups of persons were examined. The first consisted of 14 individuals with trisomy 21 Down syndrome. The second consisted of 12 persons with Williams syndrome and the deletion on chromosome 7 was confirmed in all the subjects by FISH. 32 children with typical cognitive abilities, of comparable mental age, evaluated with the L-M form of the Stanford-Binet Intelligence Scale formed the control group. The Down syndrome and Williams syndrome groups did not differ in mental age but were significantly different in their chronological age. For this reason, distinct mental-age control groups were identified for each experimental group (Down syndrome and Williams syndrome). In Table 1 , gender, means and standard deviations of chronological and mental ages for the groups included in the study are shown.

Table I. Profile of Down syndrome, Williams syndrome and typically developing mental age matched groups.

\section{Neuropsychological tests}

Consistent with our hypothesis, the neuropsychological battery included tests for evaluating implicit memory (Tower of London, Fragmented Pictures Test, Serial Reaction Time Test, Word Completion), episodic explicit memory tests for verbal material (Free Recall of a list of unrelated words) and episodic explicit memory tests for visual-perceptual material (Explicit recognition of material studied in the Fragmented Pictures Test).

The participants were tested individually. Administration of the entire protocol required two sessions of approximately one hour each, on two successive days. 


\section{Implicit Memory Tasks}

\section{Tower of London}

This test evaluates a cognitive procedure learning ability (Vicari, Bellucci \& Carlesimo, 2000) and consists of a wooden rectangular base with three perpendicular sticks of different lengths, and three balls of different colours (red, blue and green) with holes through their centres. At the start of the test, the examiner places the balls in the starting position (the green and red balls on the first stick and the blue ball on the second stick). The examiner demonstrates the first item in the test, which is a particular pattern of ball placement and the participant is asked to reproduce it, being careful to move only one ball at a time and using a set number of moves. The test continues in the same way for a total of 12 items, each of which can be repeated up to 3 times in case of error. The test was administered to each subject twice, with a one-hour interval between tests. In scoring the participants' performances, three points were given if the item was completed on the first attempt, two points for the second attempt and one for the third attempt. The final score was the sum of the points obtained on the twelve items. If their performance improved between the first and the second testing session, it was assumed that the participant was able to learn the procedure underlying the test and to use it after a time interval.

\section{Serial Reaction Time Test}

This test explores the ability to learn a visuo-motor sequence (Vicari, Bellucci \& Carlesimo, 2000). It was administered on a Compaq LTE 5280 portable computer, which controlled stimulus presentation and reaction times, the data being stored on-line. The participant sat facing the video screen on which a series of single coloured circles (green, blue or red) appeared centrally.

To perform the task the participant was instructed to watch these stimuli and press the space bar as quickly as possible every time the green circle appeared on the screen. The test consisted of 5 sequences of 45 stimuli each. In the first sequence, the colour alternation was random and therefore, unpredictable. In the next three sequences, (II, III and IV) the colour alternation was strictly ordered (red, blue and green). In the final sequence (V) the order was again random. The software automatically recorded the time between the appearance of the stimulus on the screen and the participant's response (reaction time). If the participant had learned the order in which the colours alternated on the screen, then the reaction times in the ordered sequences would gradually be reduced with respect to the first random sequence, and more importantly would worsen drastically during the last random sequence.

\section{Fragmented Pictures Test}

This is a repetition-priming test for visual material (Vicari, Bellucci \& Carlesimo, 2000). We used two sets of 7 stimulus drawings of common objects selected from the Gollin series. For each drawing, we made a set of six other pictures, each with a different level of fragmentation: the first had only a few pieces of the figure, while the seventh showed the complete figure. The two sets of drawings were homogeneous for level of fragmentation at which the pictures could be identified, as revealed by performances of 20 typically developing children to which the test was preliminarily administered.

At the beginning of the study, each participant was shown the 7 complete objects of one of the two test sets. Immediately afterwards, during the test phase, the same participant was shown the 14 series of fragmented pictures, one at a time, starting from the most fragmented and progressing to the most complete, up to the figure in which the participant recognised the stimulus. For each figure, the score given depended on the level of fragmentation at which recognition occurred. So, for example, if the picture was recognised at the most fragmented level, the score was 7. In contrast, if the figure was recognised at the most complete level, the score was 1. A priming effect was demonstrated if the previously studied pictures were recognised at a more fragmented level than pictures that had not been seen previously.

\section{Stem Completion}

This is a repetition-priming test for visually presented verbal material (Vicari, Bellucci \& Carlesimo, 2000). Thirty words of comparable frequency of use and varying in length from 4 to 11 letters were chosen. Three-letter word beginnings occurred only once among the 30 words selected and represented the beginning of at least 10 words in the Zingarelli (1983) dictionary. The 30 words were divided into two lists of 15 words each. The two lists were similar as to average frequency of use and word length. Each stimulus was printed on a single sheet of A4-sized paper. During the study phase, each participant was requested to read the 15 words on one of the two lists presented alone and to express his/her opinion about its likebility ("I like it", "I don't like it"). Each word was presented for about 5 seconds and if the participant had difficulty in reading it, the word was then read aloud by the examiner. During the test phase, which immediately followed the study phase, the 30 wordbeginnings were presented one at a time. In order to control for potential recency effects (expression of short-term memory contribution to performance in the stem completion test), the stems relative to the words in the three final positions of the study list did not occupy any of the first 5 positions in the test list.

The two lists were used equally often as target and control for each participant group. The purpose of this test is to evaluate the influence of previous exposure to a list on the successive completion test, without explicit reference to the previous study phase. Therefore, the participants were asked only to complete each word beginning with the first word that came into their mind. Also in this case, if the participants were not able to read the word beginnings by themselves, the examiner would read them. The priming effect was demonstrated if the studied words were completed more frequently than the new words. 


\section{Explicit Memory Tasks}

\section{Word-list learning:}

Each participant was given a list of 12 words of similar frequency of use that were not semantically related. The list was presented both orally (the examiner read one word at a time) and visually (each word was reproduced in a photograph) at the same time for 5 consecutive times; each time the participant was asked to immediately repeat as many items as possible. The score was the total number of words recalled by each participant in the five trials.

\section{Word Recognition:}

Ten minutes after administration of the Stem Completion Test, during which children were not engaged in any particular task, an explicit yes/no Recognition Test was given. In this test, the 15 words forming the study list in the Stem Completion were read by the examiner together with other 15 unstudied words, randomly intermixed. The children were requested to discriminate between the previously studied words from the unstudied words. Performance level was computed as the number of hit rates compared with incorrect responses.

\section{Picture Recognition:}

In this test, the 14 figures observed during the Fragmented Pictures Test (study phase and test phase) were presented one at a time; they were randomly intermixed with 14 other figures taken from the same series. The children were requested to discriminate the previously studied from the new pictures. Also in this case, hit rates and incorrect responses represented the performance score.

\section{Results}

In Table 2 results obtained by participants with Down syndrome and their controls in the implicit memory tasks are reported. In all the tasks considered, the children with Down syndrome and typical controls did not differ. In particular, a similar pattern within the two groups was observed both in the Serial Reaction Time and Tower of London test.

Concerning the Williams syndrome group, the results were quite different. Indeed, although participants with Williams syndrome were similar to typical controls in the priming repetition tasks (for verbal as well as for visual material) they failed to show the same pattern as typical controls

\begin{tabular}{|c|c|c|c|c|}
\hline & & DS & MA & $\mathbf{p}$ \\
\hline \multirow[t]{4}{*}{$\begin{array}{l}\text { Implicit } \\
\text { Memory Tasks }\end{array}$} & $\begin{array}{l}\text { Serial Reaction Time } \\
\text { (V-IV trials) }\end{array}$ & +102.7 & +162.5 & n.s \\
\hline & $\begin{array}{l}\text { Tower of London } \\
\text { (II-I testing session score) }\end{array}$ & $\begin{array}{r}M=2.8 \\
(4.5)\end{array}$ & $\begin{array}{r}M=3.2 \\
(1.9)\end{array}$ & n.s. \\
\hline & Fragmented Picture Test & $\begin{array}{c}M=3.64 \\
(5.1)\end{array}$ & $\begin{array}{r}M=6.3 \\
(3.7)\end{array}$ & n.s. \\
\hline & Word Stem Completion & $\begin{array}{r}M=4.9 \\
(2.4)\end{array}$ & $\begin{array}{c}M=5.65 \\
(2.9)\end{array}$ & n.s. \\
\hline
\end{tabular}

Table 2. Implicit memory task results for Down syndrome and typical mental age matched control groups.

\begin{tabular}{|c|c|c|c|c|}
\hline & & WS & MA & $\mathbf{p}$ \\
\hline \multirow[t]{4}{*}{$\begin{array}{l}\text { Implicit } \\
\text { Memory Tasks }\end{array}$} & $\begin{array}{l}\text { Serial Reaction Time } \\
\text { (V-IV trials) }\end{array}$ & +62.0 & +219.0 & $\mathrm{p}=.0 \mathrm{I}$ \\
\hline & $\begin{array}{l}\text { Tower of London } \\
\text { (II-I testing session score) }\end{array}$ & $\begin{array}{r}M=1.2 \\
(2.6)\end{array}$ & $\begin{array}{r}M=3.2 \\
(I .5)\end{array}$ & $\mathrm{p}<.05$ \\
\hline & Fragmented Picture Test & $\begin{array}{r}M=3.9 \\
(2.9)\end{array}$ & $\begin{array}{r}M=4.8 \\
(3.2)\end{array}$ & n.s. \\
\hline & Word Stem Completion & $\begin{aligned} & M= 5.3 \\
&(3.7)\end{aligned}$ & $\begin{array}{r}M=5.9 \\
(2.5)\end{array}$ & n.s. \\
\hline
\end{tabular}

Table 3. Implicit memory task results for Williams syndrome and typical mental age matched control groups.

\begin{tabular}{llrrr} 
& & & & \\
& & DS & MA & P \\
\hline Explicit & Free recall & $M=35.0$ & $M=45.3$ & $\mathrm{p}<.00 \mathrm{I}$ \\
Memory Tasks & & $(8.2)$ & $(6.4)$ & \\
& Words rec. & $\mathrm{M}=25.6$ & $\mathrm{M}=29.0$ & $\mathrm{p}<.05$ \\
& & $(5.2)$ & $(\mathrm{I} . \mathrm{I})$ & \\
& Pictures rec. & $\mathrm{M}=24.8$ & $\mathrm{M}=28.0$ & $\mathrm{p}<.0 \mathrm{I}$ \\
& & $(5.4)$ & $(0.0)$ &
\end{tabular}

Table 4. Explicit memory task results for Down syndrome and typical mental age matched control groups.

\begin{tabular}{llccc} 
& & WS & MA & p \\
\hline Explicit & Free recall & $M=42.9$ & $M=47.1$ & n.s. \\
Memory Tasks & & $(6.8)$ & $(6.6)$ & \\
& Words rec. & $M=28.7$ & $M=29.2$ & n.s. \\
& & $(1.05)$ & $(1.03)$ & \\
& Pictures rec. & $M=27.3$ & $M=28.0$ & n.s. \\
& & $(1.02)$ & $(0.0)$ & \\
& & & \\
& & &
\end{tabular}

Table 5. Explicit memory task results for Williams syndrome and typical mental age matched control groups.

both in the Serial Reaction Time and Tower of London test (Table 3).

On the explicit memory task, participants with Down syndrome are always poorer than typical controls (Table 4).

On the other hand the performances of participants with Williams syndrome did not differ from those of typical controls (Table 5). 


\section{Discussion}

The principal result of our study was the demonstration of distinct memory patterns in persons with Down syndrome compared with Williams syndrome. With regard to explicit memory abilities, participants with Williams syndrome showed a similar performance profile to the typically developing mental-age matches. In contrast, participants with Down syndrome obtained lower performance scores than the other two groups. In the implicit memory domain, although we observed comparable results between the two experimental groups in repetition priming tasks, Williams syndrome participants were impaired in the ability to learn new procedures.

The discrepant performance profiles exhibited by children with Down syndrome and Williams syndrome suggest that the procedural learning deficit exhibited by Williams syndrome (as well as the deficit in explicit memory of Down syndrome) is not an expression of the global cognitive impairment affecting people with intellectual disabilities but, rather, that it is a peculiarity of the Williams syndrome group. It presumably results from some specific characteristics of their anomalous brain development. Concerning our study, any attempt to identify which neuroanatomical structure is specifically involved in the implicit memory impairment displayed by the participants with Williams syndrome must necessarily be based on qualitative analogies of their deficit with that displayed by adult neurological patients such as those with Huntington's disease (Willingham \& Koroshetz, 1993) in which a degenerative loss of neurons in the basal ganglia, and cerebellum are found (Molinari, Leggio \& Solida, 1997).

The brain development of children with Williams syndrome is characterised by both a remarkable atrophy of the basal ganglia (Jernigan \& Bellugi, 1990) and by a neurochemical alteration (reduction of the neurotransmitter $\mathrm{N}$-acetylaspartate) in the cerebellum (Rae, Karmiloff-Smith \& Lee, 1998), thus suggesting a neurobiological substrate for the impaired maturation of procedural learning.

In our opinion, there are two reasons for attributing a causal role to the volumetric reduction of basal ganglia in this group of people. First, the performance profile exhibited by children with Williams syndrome resembles Huntington's disease patients more than patients with cerebellor damage. Second, individuals with Down syndrome, despite atrophy of the cerebellum, show typical procedural learning of both visuo-motor and cognitive tasks, thus undermining the role of cerebellar circuit maturation in the development of skills learning. Further studies, directly evaluating the possible correlation between morphovolumetric and spectroscopic indexes of brain functioning and the ability of individuals with Williams syndrome to learn visuo-motor and cognitive procedures, are needed to better understand the relative contribution of the basal ganglia and abnormal cerebellar development in the impaired maturation of procedural memory in these persons.

\section{Acknowledgements}

Preparation of this article was supported by the Italian National Department of Health, grant n. 96/02/N/002.

Part of the results presented here were already published in Neuropsychologia, 2000, 38, 240-251.

I gratefully acknowledge Giovanni Augusto Carlesimo and Samantha Bellucci's contributions to this study. Also, I am grateful to AIPD (Italian Association of Persons with Down's Syndrome) and Don Guanella Institute for their friendly cooperation. Many thanks are due to the children who participated in the study as well as their parents.

\section{References}

Botta, A., Sangiuolo, F., Calza, L., Giardino, L., Potensa, S., Novelli, G., \& Dallapiccola, B. (1999) Expression analysis and protein localization of the human hpc-l/syntaxin la, a gene depleted in Williams syndrome. Genomics, 15, 525-528.

Carlesimo, G.A., Marotta, L. \& Vicari, S. (1997). Long-term memory in mental retardation: Evidence for a specific impairment in subjects with Down's syndrome. Neuropsychologia, 35, 1, 71-79.

Ewart, A.K., Morris, C.A. \& Atkinson, D. (1993). Hemizygosity at the elastin locus in a developmental disorders, Williams syndrome. Nature Genetics, 5, 11-16.

Frangiskakis, J.M., Ewart, A.K. \& Morris, C.A. (1996). LIM-Kinase-1 hemizygosity implicated in impaired visuospatial constructive cognition. Cell, 86, 59-69.

Hulme, C. \& Mackenzie, S. (1992). Working Memory and Severe Learning Difficulties. Hove: Erlbaum.

Jernigan, T.J. \& Bellugi, U. (1990). Anomalous brain morphology on magnetic resonance images in Williams syndrome. Archives of Neurology, 47, 529-533.

Miller, J.F. (1992). Development of speech and language in children with Down syndrome. In I.T. Lott \& E.E. McCoy (Eds), Down Syndrome, (pp. 39-50). New York: Wiley-Liss.

Miller, J.F. (1988). The developmental asynchrony of language development in children with Down syndrome. In L. Nadel (Ed), Psychobiology of Down's Syndrome, (pp.167-198). Cambridge, MA: MIT Press.

Molinari, M., Leggio, M.G. \& Solida, A. (1997). Cerebellum and procedural learning: evidence from focal cerebellar lesions. Brain, 120, 1753-1762.

Pezzini, G., Vicari, S., Volterra, V., Milani, L. \& Ossella, M.T. (1999). Children with Williams syndrome: Is there a single neuropsychological profile? Developmental neuropsychology, 15 (1), 141-155.

Rae, C., Karmiloff-Smith, A. \& Lee, M.A. (1998). Brain biochemistry in Williams syndrome. Evidence for a role of the cerebellum in cognition. Neurology, 51, 33-40.

Tassabehji, M.K., Karmiloff-Smith, A. \& Grant, J. (1998). Genotype phenotype correlations in Williams syndrome. American Journal of Human Genetic, 61, 11.

Tassabehji, M.K., Metcalfe, K. \& Fergusson, W.D. (1996). LIM-Kinase-1 detected in Williams Syndrome. Nature Genetics, 13, 272-273. 
Vicari, S., Albertini, G. \& Caltagirone, C. (1992). Cognitive Profiles in Adolescents with Mental Retardation. Journal of Intellectual Deficiency Research, 36, 415-423.

Vicari, S., Bellucci, S. \& Carlesimo, G.A. (2000). Implicit and explicit memory: a functional dissociation in persons with Down syndrome. Neuropsychologia, 38, 240-251.

Vicari, S., Carlesimo, G.A., Brizzolara, D. \& Pezzini, G. (1996). Short-term memory in children with Williams syndrome: a reduced contribution of lexical- semantic knowledge to word span. Neuropsychologia, 34(9), 919-925.

Vicari, S., Caselli, M.C. \& Tonucci, F. (2000). Early language development in Italian children with Down syndrome: asynchrony of lexical and morphosyntactic abilities. Neuropsychologia, 38, 634-644.
Volterra, V., Capirci, O., Pezzini, G., Sabbadini, L. \& Vicari, S. (1996). Linguistic abilities in Italian Children with Williams Syndrome. Cortex, 32, 663-677.

Willingham, D.B. \& Koroshetz, W.J. (1993). Evidence for dissociable motor skills in Huntington's disease patients. Psychobiology, 21, 173-182.

Zingarelli, N. (1983) Vocabolario Della Lingua Italiana. Milano: Zanchelli. 